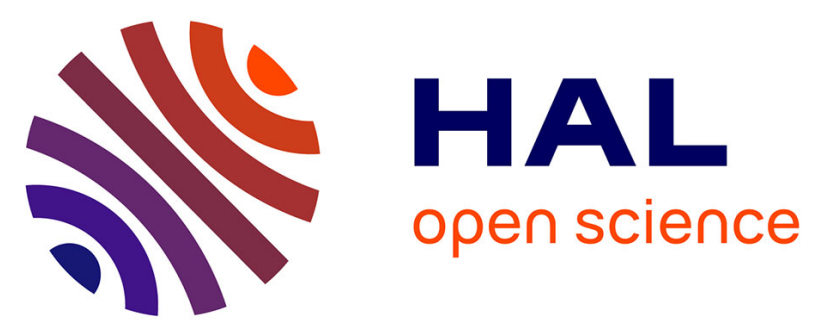

\title{
Electromagnetic Transient Analysis of Transmission line based on rational Krylov approximation
}

\author{
Damien Tromeur Dervout, Amjad Mouhaidali, Damien Tromeur-Dervout, \\ Olivier Chadebec, Jean-Michel Guichon, Sebastien Silvant
}

\section{- To cite this version:}

Damien Tromeur Dervout, Amjad Mouhaidali, Damien Tromeur-Dervout, Olivier Chadebec, JeanMichel Guichon, et al.. Electromagnetic Transient Analysis of Transmission line based on rational Krylov approximation. IEEE Transactions on Power Delivery, 2021, 36 (5), pp.2913-2920. 10.1109/TPWRD.2020.3029676 . hal-02995214

\section{HAL Id: hal-02995214 \\ https://hal.science/hal-02995214}

Submitted on 9 Nov 2020

HAL is a multi-disciplinary open access archive for the deposit and dissemination of scientific research documents, whether they are published or not. The documents may come from teaching and research institutions in France or abroad, or from public or private research centers.
L'archive ouverte pluridisciplinaire HAL, est destinée au dépôt et à la diffusion de documents scientifiques de niveau recherche, publiés ou non, émanant des établissements d'enseignement et de recherche français ou étrangers, des laboratoires publics ou privés. 


\title{
Electromagnetic Transient Analysis of Transmission line based on rational Krylov approximation
}

\author{
Amjad Mouhaidali, Damien Tromeur-Dervout, Olivier Chadebec, Jean-Michel Guichon, and Sebastien Silvant
}

\begin{abstract}
This paper shows a comparison between Vector fitting and rational Krylov fitting techniques for the determination of rational models concerning the fitting accuracy, the computational performances and the model order. Primarily, the mathematics behind the second technique are presented. It should be noted that rational Krylov fitting have never been used in transmission line modeling. A new procedure is proposed to use rational Krylov fitting instead of vector fitting in the universal line model (ULM). Furthermore, it is demonstrated that this procedure has several advantages over the traditional one. Two illustrative examples involving a transmission system are presented for validation of the new procedure.
\end{abstract}

Index Terms-Electromagnetic transient, frequency dependency, rational krylov approximation,Vector fitting, transmission line model, passivity.

\section{INTRODUCTION}

In the frequency domain and for electromagnetic transient studies, rational approximation is used to reproduce a given frequency behavior. This approximation leads to recursive convolution and fast computations in the time domain [1]. Examples of applications are the modeling of transmission lines and cables [2], power transformers at high frequencies and the Frequency-Dependent Network Equivalents (FDNE) [3]. A general rational representation of a frequency dependent function $f(s)$ has the form:

$$
f(s) \approx r(s)=\sum_{m=1}^{N} \frac{c_{m}}{s-q_{m}}+d
$$

where $s=j \omega$ in $\mathrm{rad} / \mathrm{s}$, the model order $\mathrm{N}$; the poles $q_{m}$ and residues $c_{m}$ are either real or come in complex conjugate pairs; $d$ is a constant. In the case of cable modeling, the matrix $f$ corresponds to the frequency dependent characteristic admittance $Y_{c}(s)$ or the propagation function $H(s)$.

The vector fitting VFIT [4], [5] technique is widely used by the engineering community for the achievement of rational models. VFIT has a relatively simple formulation, high computational efficiency and delivers high model accuracy. Recently, VFIT was combined with frequency partitioning and model order reduction via balanced realizations to approximate FDNE [6] or transmission lines and cables [7]. A novel approach is proposed in [8], where the Matrix Pencil Method (MPM) and the Loewner Matrix (LM) are incorporated into the VFIT technique.

A. Mouhaidali, D. Tromeur-Dervout and S. Silvant are with SuperGrid Institute, 23 rue Cyprian, 69628 Villeurbanne Cedex, France.

D. Tromeur-Dervout is with Univ Lyon, Universit Claude Bernard Lyon 1, CNRS UMR 5208, Institut Camille Jordan, F-69622 Villeurbanne, France.

O. Chadebec and J-M. Guichon are with Univ. Grenoble Alpes, CNRS, Grenoble INP, G2Elab, 38000 Grenoble, France.
A new rational approximation method has emerged recently and it is based on the Rational Kylov fitting RKFIT [9]. This method is based on Rational Arnoldi Decomposition (RAD) for poles relocation. The used orthonormal RADs deliver a well-conditioned basis for the numerical linear algebra problems, and a more robust and faster convergent algorithm. The main difference between VFIT and RKFIT is the poles relocation method. Both methods solve a Least Square (LS) problem at each iteration, however VFIT uses a projection space represented in the partial fraction basis and RKFIT uses a projection space represented in discrete-orthogonal rational functions.

In this paper, the advantages of RKFIT are highlighted regarding the automatic determination of the model order $(N)$ with a built-in model order reduction technique, the fitting accuracy and the pole relocation convergence. It is proposed to use RKFIT in the implementation of the Universal Line Model (ULM) [2] instead of VFIT.

Unfortunately, RKFIT cannot guarantee the passivity of the model. If passivity violation is detected, post fitting passivity algorithms are necessary. Various passivity enforcement technique are presented in [10].

This paper is organized as follows. The rational Krylov algorithm is presented in Section II. Section III assess the numerical performances through different examples. Section IV demonstrate the application of the new approach for a single overhead line and an underground cable system.

\section{RATIONAL KRYLOV FITTING ALGORITHM}

\section{A. Mathematical background of RKFIT}

RKFIT is an iterative algorithm for solving nonlinear weighted rational least square problems [9]. Let's consider The following matrices and vector:

- $A=\operatorname{diag}\left(s_{i}\right) \in \mathbb{C}^{N_{s}, N_{s}}$, where $N_{s}$ is the number of frequency samples

- $F=\operatorname{diag}\left(f_{i}\right) \in \mathbb{C}^{N_{s}, N_{s}}$ with $f_{i}=f\left(s_{i}\right)$

- $b \in \mathbb{C}^{N_{s}}$, where $b$ is the starting vector.

The algorithm tries to approximate $F \mathbf{b}$ as $r(A) \mathbf{b}$, where $r$ is a rational function of the form:

$$
r=\frac{p_{m+k}}{q_{m}}
$$

where $p_{m+k}$ and $q_{m}$ are polynomials of degree less or equal to $m+k$ and $m$ respectively such that the relative error $\varepsilon_{e r}$ is minimal:

$$
\varepsilon_{e r}=\frac{\|F b-r(A) b\|_{2}}{\|F b\|_{2}} \rightarrow \min
$$


The RKFIT algorithm is extended to approximate a family of matrices all sharing a common denominator $q_{m}$ [9]. This feature fits all the element of $Y_{c}(s)$ with a common pole set.

The rational Krylov fitting is a fitting method based on Krylov space. We will provide a brief overview of the Krylov methods. A more exhaustive analysis can be found in [11], [12]. Given the matrix $A$ and the vector $b$, the corresponding order $-m$ Krylov subspace is defined as:

$$
\mathcal{K}_{m+1}(A, b) \stackrel{\text { def }}{=} \operatorname{span}\left\{b, A b, \ldots, A^{m} b\right\}
$$

The starting point in the RKFIT algorithm is to consider a non zero polynomial $q_{m} \in P_{m}$ that has no roots in $\Lambda(A)$, the set of eigenvalues of $A$. The rational Krylov space of order $m$ associated with $\left(A, b, q_{m}\right)$ is defined as:

$$
\mathcal{Q}_{m+1}\left(A, b, q_{m}\right) \stackrel{\text { def }}{=} q_{m}(A)^{-1} \mathcal{K}_{m+1}(A, b)
$$

To extract information out of the rational Krylov space, the first step is to construct the rational Arnoldi decomposition of the space $\mathcal{Q}_{m+1}\left(A, b, q_{m}\right)$ as follows:

$$
A V_{m+1} \underline{K_{m}}=V_{m+1} \underline{H_{m}}
$$

where the columns of $V_{m+1}$ are an orthogonal basis of $\mathcal{Q}_{m+1}\left(A, b, q_{m}\right) . \quad K_{m}$ and $\underline{H_{m}}$ are unreduced upper-

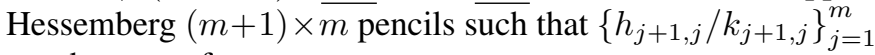
are the root of $q_{m}$.

The rational approximation associated with the rational Arnoldi decomposition in (6) satisfies:

$$
r(A) b=\left(V_{m+1} \underline{K_{m}}\right) f\left(\underline{K_{m}^{\dagger}} \underline{H_{m}}\right)\left(V_{m+1} \underline{K_{m}}\right)^{\dagger} b
$$

\section{B. Pole relocation process}

The pole relocation process aims to find new poles that would provide a better approximation to $F b$ from previous ones. Two linear spaces in $\mathbb{C}^{N_{c}}$ are used, where the search space is $\mathcal{S}=\mathcal{R}\left(V_{m+1}\right)=\mathcal{Q}_{m+1}\left(A, b, q_{m}\right)$ and the target space $\mathcal{T}=\mathcal{R}\left(\hat{V}_{m+k+1}\right)=\mathcal{Q}_{m+k+1}\left(A, b, q_{m}\right)$. Let's consider the orthogonal projection onto $\mathcal{T}$ as $P_{\mathcal{T}}=\hat{V}_{m+k+1} \hat{V}_{m+k+1}^{*}$. Then we can write that $r(A) b$ should be the orthogonal projection of $F b$ onto the target space $\mathcal{T}$ :

$$
\begin{aligned}
\|F b-r(A) b\|_{2} & =\left\|F b-\hat{V}_{m+k+1} \hat{V}_{m+k+1}^{*} F b\right\|_{2} \\
& =\left\|\left(I-P_{\mathcal{T}}\right) F b\right\|_{2}
\end{aligned}
$$

Then we search for vector $v \in \mathcal{S}$ such that:

$$
v=\underset{\substack{\bar{v} \in \mathcal{S} \\\|\bar{v}\|_{2}=1}}{\operatorname{argmin}}\left\|\left(I-P_{\mathcal{T}}\right) F \bar{v}\right\|_{2}
$$

Defining the matrix $S=F V_{m+1}-\hat{V}_{m+k+1}\left(\hat{V}_{m+k+1}^{*} F V_{m+1}\right)$, a solution is given by $v=V_{m+1} \hat{c}$, where $\hat{c}$ is the right singular vector of $S$ associated to the smallest singular value $\sigma_{\min }$. Let a nonzero polynomial $\bar{q}_{m} \in P_{m}$ that has no roots in $\Lambda(A)$, then:

$$
\mathcal{Q}_{m+1}\left(A, b, q_{m}\right)=\mathcal{Q}_{m+1}\left(A, \bar{q}_{m}(A) q_{m}(A)^{-1} b, \bar{q}_{m}\right)
$$

The pole relocation consists in finding the polynomial $\bar{q}_{m}$ such that:

$$
v=V_{m+1} c=\bar{q}_{m}(A) q_{m}(A)^{-1} b
$$

\section{Partial fraction basis calculation}

The approximant $\mathbf{r}$ is calculated via a coefficient vector $\mathbf{c} \in$ $\mathbb{C}^{m+k+1}$ such that:

$$
\mathbf{c}=\frac{\hat{V}_{m+k+1}^{*}(F b)}{\|b\|_{2}}
$$

The computation of lead to the evaluation of the relative error and compared it to the desired approximation tolerance. The full development of the rational Krylov fitting process is proposed in [9], [13]. The high-level description of RKFIT is presented in Fig 1.

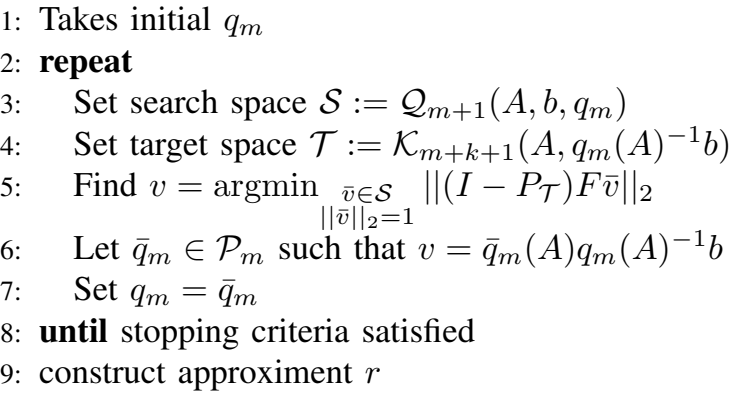

\begin{tabular}{|c|c|c|c|}
\hline \multirow{2}{*}{ Example } & \multirow{2}{*}{ Model order } & \multicolumn{2}{|c|}{ RMS error } \\
\hline & & VFIT & RKFIT \\
\hline Matrix fitting & 10 & $1.139 \times 10^{-2}$ & $6.246 \times 10^{-3}$ \\
\hline Toolbox [15] & 20 & $6.069 \times 10^{-3}$ & $1.233 \times 10^{-3}$ \\
\hline \multirow{2}{*}{$\begin{array}{l}\text { CD player SLICOT } \\
\text { benchmark [16] }\end{array}$} & 10 & $1.683 \times 10^{1}$ & $3.675 \times 10^{0}$ \\
\hline & 20 & $1.674 \times 10^{1}$ & $9.061 \times 10^{-3}$ \\
\hline \multirow{2}{*}{$\begin{array}{l}\text { ISS model SLICOT } \\
\text { benchmark [16] }\end{array}$} & 10 & $6.729 \times 10^{-4}$ & $8.735 \times 10^{-5}$ \\
\hline & 20 & $3.016 \times 10^{-4}$ & $1.507 \times 10^{-5}$ \\
\hline \multirow{2}{*}{$\begin{array}{l}\text { Nonlinear eigenvalue, } \\
\text { buckling example [17] }\end{array}$} & 10 & $1.476 \times 10^{-4}$ & $2.292 \times 10^{-10}$ \\
\hline & 20 & $1.270 \times 10^{-11}$ & $2.389 \times 10^{-12}$ \\
\hline \multirow{2}{*}{$\begin{array}{c}\text { ISS model with } \\
\text { additive normally } \\
\text { distributed noise [16] }\end{array}$} & 10 & $5.557 \times 10^{-1}$ & $4.702 \times 10^{-2}$ \\
\hline & 20 & $6.280 \times 10^{-2}$ & $3.798 \times 10^{-2}$ \\
\hline
\end{tabular}

Fig. 1. High-level description of RKFIT

\section{ACCURACY COMPARISON OF RKFIT AND VFIT}

In [14] a selection of algorithms for rational approximation are discussed. Table I recapitulates the main RMS error values for various example and different model order studied in [14]. It was shown that RKFIT outperforms VFIT for rational approximation regarding the approximation accuracy.

TABLE I

RMS ERROR VALUES FOR ALL TESTED EXAMPLES IN [14]

In this section, we will focus on the main differences of the two methods. First the model order determination, then the accuracy and pole convergence with weight, after that the choice of the starting poles and at the end the system passivity. An example from the literature used as benchmark for transmission line studies will be fitted with both methods. This case study is taken from [5] and represent the $6 \times 6$ 
admittance matrix of the distribution network of Fig. 2 measured from nodes $\mathbf{A}$ and $\mathbf{B}$. The magnitude of the elements of the admittance matrix are plotted in Fig.3. We choose to fit only the first column of the admittance matrix. This case is challenging due to multiple resonance peaks. The fitting accuracy is examined by comparing the fitting RMS error calculated as:

$$
R M S_{\text {error }}=\sqrt{\frac{\sum_{k=1}^{N_{s}} \sum_{n=1}^{N_{c}}\left|f_{n}^{\text {fitted }}\left(s_{k}\right)-f_{n}^{\text {accurate }}\left(s_{k}\right)\right|^{2}}{N_{s} N_{c}}}
$$

The Pole relocation process can be monitored by computing so-called Hausdorff distance between the pole sets at two consecutive iterations [10]. The simulations are performed using a 16 GB RAM computer, i5-9600 3.10 GHz-processor with MATLAB 2019a.

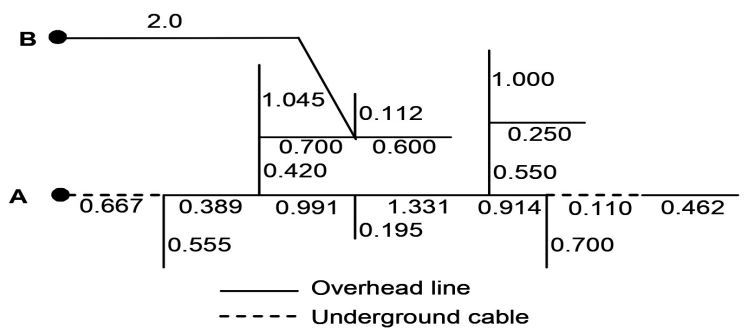

Fig. 2. Power system distribution system

The element of the vector are fitted using the relaxed nontriviality constraint of VFIT, provided in the function vectfit3.m, and the unstable poles are forced to be stable by flipping them.

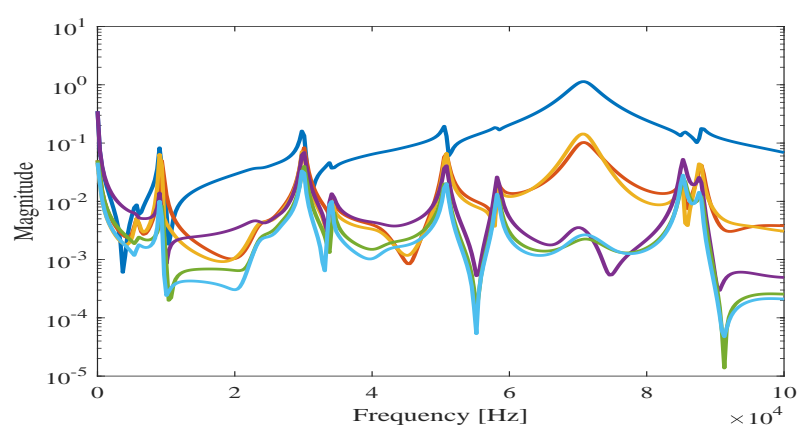

Fig. 3. Magnitude of admittance matrix of the power system

\section{A. Model order determination and reduction technique}

In the first iteration of RKFIT, the model order is chosen to be smaller than the number of frequency samples and high enough to approximate the function with a low deviation error. When RKFIT founds an adequately good approximation, it reduces the model order and tries not to degrade the approximation accuracy. The admittance matrix is fitted using RKFIT with reduction technique. The approximation error is presented in Figure 4 for two error tolerances. The maximal model order is fixed to 70 and the starting poles is chosen to be infinite.

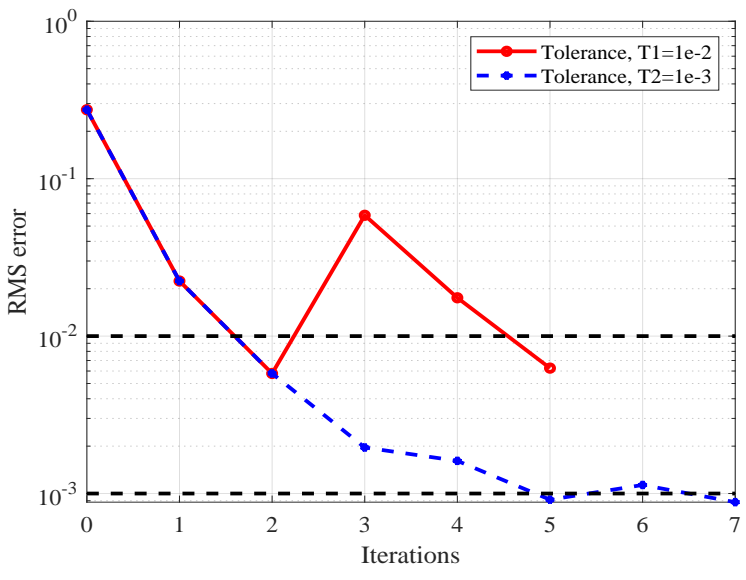

Fig. 4. Model order reduction with RKFIT

RKFIT achieve an RMS error below the tolerance at iterations 2 and 5, after which the degree reduction takes place. The denominator degree is reduced to $N=33$ and $N=49$ for tolerances $T 1$ and $T 2$ respectively. After 1 or 2 iterations of pole relocation, RKFIT reaches the fixed tolerance and the algorithm is stopped.

\section{B. Fitting accuracy and pole convergence}

In order to compare the fitting accuracy, The model order is fixed for RKFIT and VFIT. The fitting is done with three weighting schemes (no weight, strong inverse magnitude weight, and weak inverse magnitude weight). The weight is common for all the vectors elements. The initial poles are chosen to be complex and linearly spaced.

TABLE II

RELATIVE APPROXIMATION ERROR OF VFIT AND RKFIT

\begin{tabular}{llccc}
\hline & \multicolumn{1}{c}{ Weight } & Order & RMS error & CPU \\
\hline \multirow{4}{*}{ VFIT } & No weight & 50 & $1.58 \times 10^{-4}$ & $0.87 \mathrm{~s}$ \\
\cline { 2 - 5 } & Strong inverse & 50 & $1.00 \times 10^{-3}$ & $0.85 \mathrm{~s}$ \\
& magnitude weight & 70 & $4.43 \times 10^{-7}$ & $1.14 \mathrm{~s}$ \\
\cline { 2 - 5 } & Weak inverse & 50 & $2.65 \times 10^{-4}$ & $0.78 \mathrm{~s}$ \\
& magnitude weight & 70 & $1.19 \times 10^{-8}$ & $1.16 \mathrm{~s}$ \\
\hline \multirow{3}{*}{ RKFIT } & No weight & 50 & $9.88 \times 10^{-5}$ & $1.04 \mathrm{~s}$ \\
\cline { 2 - 5 } & Strong inverse & 50 & $3.18 \times 10^{-4}$ & $1.00 \mathrm{~s}$ \\
& magnitude weight & 70 & $3.08 \times 10^{-8}$ & $1.45 \mathrm{~s}$ \\
\cline { 2 - 5 } & Weak inverse & 50 & $2.02 \times 10^{-4}$ & $1.06 \mathrm{~s}$ \\
& magnitude weight & 70 & $1.51 \times 10^{-8}$ & $1.44 \mathrm{~s}$ \\
\hline
\end{tabular}

The RMS error obtained by fitting the admittance matrix through both methods for different fitting orders and weighting schemes is given in Table II. This table validates that RKFIT outperforms VFIT for different weighting schemes and orders regarding fitting accuracy except with the case with weak inverse magnitude weight and high model order. However, VFIT is slightly faster.

The convergence of the pole relocation process as function of the iteration count is shown in Fig.5. The model order is 


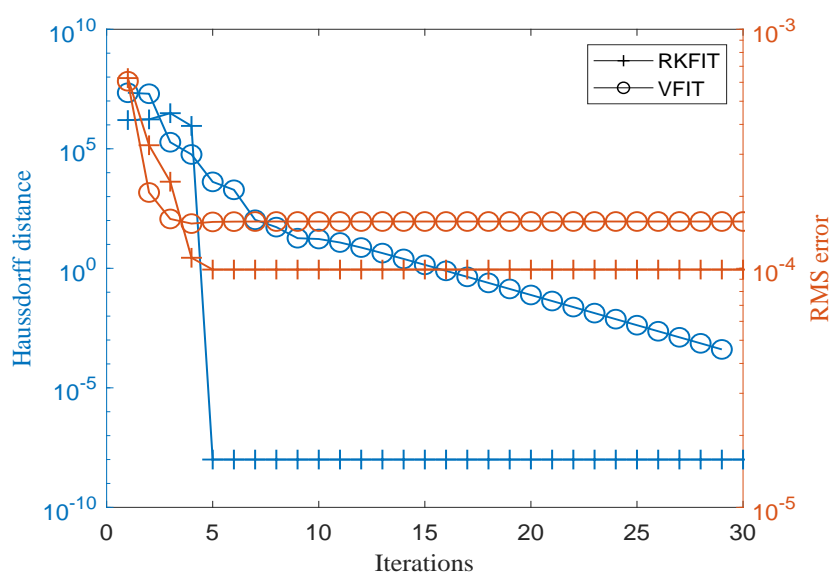

Fig. 5. Convergence of pole relocation process by RKFIT against VFIT

$N=50$ and no weight scheme is applied. The Haussdorf distance is presented on the left side of the graph and the RMS error on the right side. It is chosen to restrict the lowest distance to $10^{-8}$ for illustration purpose. This graph shows that RKFIT converges faster than VFIT. After 5 iterations RKFIT reaches the set of poles that delivers the smallest RMS error and the distance becomes zero. For RKFIT, it is remarked the correlation between the distance and the RMS error. When the distance becomes zero, the RMS is at the lowest value. However for VFIT, the RMS error reaches the lowest value when the distance starts to decrease linearly. This observation can be used as the fitting stopping criteria.



Fig. 6. Convergence fo pole relocation process by RKFIT and VFIT

For the same previous example, if the model order is increased to $N=100$, VFIT does not converge. Fig. 6 shows the convergence of the pole relocation process. RKFIT converges within 4 iterations while VFIT diverges, the Haussdorf distance rises linearly and the RMS error oscillates.

\section{Choice of the starting poles}

One of the main advantages of RKFIT is that the user requires no a priori knowledge of the initial poles location and distribution. Poles can be chosen to be infinite and RKFIT will relocate them. Fig. 7 shows the convergence of the pole relocations for different initial pole distributions. This figure demonstrates that RKFIT converges faster than VFIT for different starting poles. It should be noted that the infinite value is fixed by the ieee arithmetic representation for positive infinity in [18].



Fig. 7. Convergence of poles relocation process with different starting poles

\section{D. passivity}

For a multi-port electrical network, the passivity is guaranteed if and only if its transfer admittance matrix $Y_{n}(s)$ has a purely positive real part through the whole frequency range [10]. $Y_{n}(s)$ is positive definite if and only if its Hermitian part $Y_{H}(s)$ has only positive eigenvalues:

$$
\operatorname{eig}\left(Y_{H}(s)\right)=\operatorname{eig}\left(Y_{n}(s)+Y_{n}^{*}(s)\right)
$$

where $Y_{n}^{T}(s)$ is the conjugate transpose of $Y_{n}$. The admittance matrix is fitted with different model order to assess the passivity violation for RKFIT and VFIT. The model's passivity proprieties are assessed using the half singularity test developed in [19] via the main function driver RPdriver.m in [15].

TABLE III

PASSIVITY ASSESSMENT FOR DIFFERENT MODEL ORDER APPROXIMATION

\begin{tabular}{lccc}
\hline Method & Model order & Passivity violation & Max passivity violation \\
\hline \multirow{2}{*}{ RKFIT } & 50 & No & None \\
\cline { 2 - 4 } & 70 & No & None \\
\cline { 2 - 4 } & 90 & Yes & -0.0005 \\
\hline \multirow{2}{*}{ VFIT } & 50 & Yes & $-8.63 \times 10^{-5}$ \\
\cline { 2 - 4 } & 70 & No & None \\
\cline { 2 - 4 } & 90 & Yes & -0.0015 \\
\hline
\end{tabular}

Table III illustrates the passivity assessment for different model order approximation. It is shown that RKFIT can deliver passive model for relatively optimal model order, However, for high order models passivity violation appears. Despite the fact that passivity violation cannot be anticipated, several passivity enforcement algorithm can solve this issue. 


\section{UNIVERSAL LINE MODEL BASED ON RKFIT}

In the ULM [2], the propagation function $H$ and the characteristic admittance $Y_{c}$ are approximated using VFIT [4]. The rational approximations are used for time domain implementation in EMTP-type simulation tools [20]. As outlined in the previous section, RKFIT gives more accurate results than VFIT. Accordingly, a fitting procedure for $Y_{c}$ and $H$ is proposed in this part using RKFIT instead of VFIT.

The proposed fitting approach has several advantages over the fitting approach implemented in the EMT-softwares:

1) The proposed approach delivers accurate fitting results with low RMS error and guarantees convergence of the algorithm.

2) The model order is automatically calculated by the RKFIT algorithm. The user only defines the RMS fitting error tolerance.

3) The user requires no a priori knowledge of the pole location. The poles can be equal to the ieee arithmetic representation for positive infinity, represented in Matlab by the value Inf.

\section{A. Rational approximation in the ULM with RKFIT}

1) Fitting the characteristic admittance: The characteristic admittance $Y_{c}$ is easily fitted since it doesn't have resonance peaks. All the elements of $Y_{c}$ are fitted with the same set of poles expressed in the following form:

$$
Y_{c}(s) \approx Y_{c_{f i t t e d}}=\sum_{m=1}^{N} \frac{c_{m}}{s-q_{m}}+D
$$

2) Fitting the propagation function: The approximation of the propagation function is more difficult since its elements contain propagation delays. The modal contributions of $H$ are calculated and a proper delay is assigned to each mode. The delay is calculated using the optimization technique proposed in [21]. The criterion proposed in [22] is used to group repetitive or close delays. Time delays are removed from the modal components and then fitted using RKFIT. The propagation function is then fitted using the poles from the modal approximation. The only remaining unknowns are the residues, and $H$ is approximated as:

$$
H(s) \approx H_{\text {fitted }}=\sum_{i=1}^{K}\left(\sum_{m=1}^{N_{i}} \frac{R_{m, i}}{s-a_{m, i}}\right) e^{\left(-s \tau_{i}\right)}
$$

where $K$ is the number of grouped modes, $\tau_{i}$ are the collapsed time delay, $R_{m, i}$ is the matrix of residues calculated for the corresponding poles $a_{m, i}$ of the grouped modes and $H_{\text {fitted }}$ stands for the rational approximation of $H$ considering the grouped modes.

Time domain implementation is based on the equation developed in [23] to reduce integration and interpolations errors.

\section{B. Numerical Results}

The proposed method is used with different transmission line configurations and the calculated results are compared with the corresponding results from VFIT. In all cases, the characteristic admittance and the propagation function matrices are calculated for the frequency range from $0.01 \mathrm{~Hz}$ up to $1 \mathrm{MHz}$ with a sampling rate of logarithmically spaced 10 points/decade.

1) Overhead Transmission Line: Let's consider a $30 \mathrm{~km}$ single conductor over-head line over a lossy ground as shown in Fig. 8 and presented in [21].

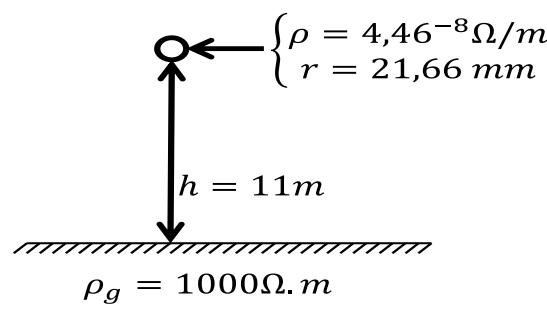

Fig. 8. single conductor overhead line

For the fitting of $Y_{c}$ and $H$, the initial poles are chosen to be infinite and the maximum allowable RMS-error is $10^{-3}$.

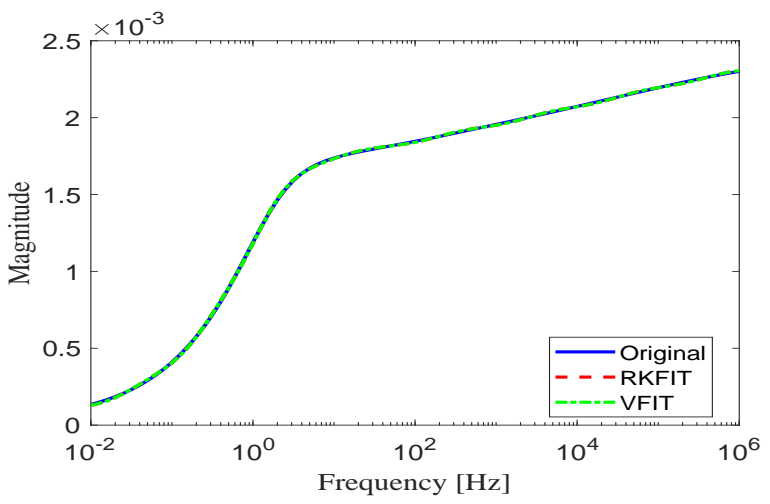

Fig. 9. Fitting of $Y_{c}$ using RKFIT and VFIT

The magnitude function of $Y_{c}$ is shown in Fig. 9. Fig 10 reports the RMS-error from the application of both methods. It is seen that RKFIT and VFIT reach the same RMS-error, however RKFIT converges faster. It is worth mentioning that both methods have the same number of poles at the end.

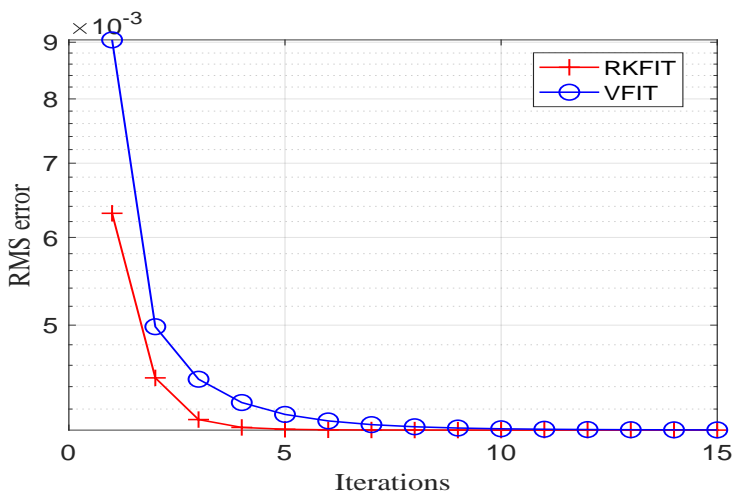

Fig. 10. RMS error as function of iteration number

As for the propagation function, the time delay is $\tau=$ $1.039 \times 10^{-4} \mathrm{sec}$. The magnitude of $H$ is plotted in Fig 11. The 
use of the proposed approach reduces the RMS error compared to VFIT and it can be seen on the magnitude plote specially at high frequencies.

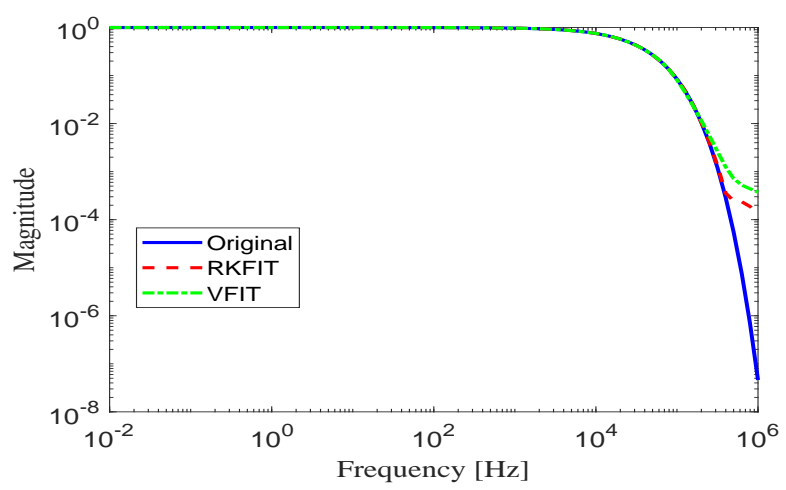

Fig. 11. Magnitude of the propagation function

2) Underground cable system : Let's consider the cable system in Fig. 12 with parameters given in Table IV. The resulting order for the fitting of $Y_{c}$ is $N=9$ for RKFIT and $N=12$ for VFIT. Fig. 13 shows the rms error as a function of the iteration number. It is seen that RKFIT gives a faster convergence and a lower order of approximation, although the final result is quite similar.

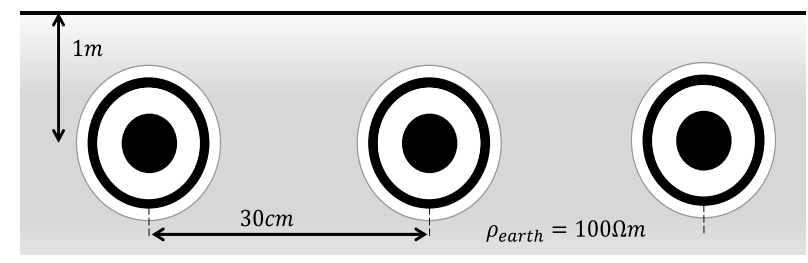

Fig. 12. Cross section of three phase $145 \mathrm{kV}$ underground cable

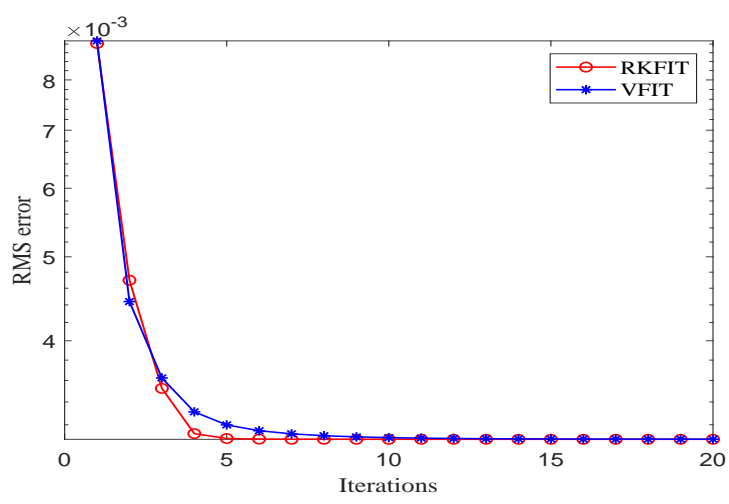

Fig. 13. RMS error as function of iteration number

TABLE IV

GEOMETRICAL AND MATERIAL PARAMETERS OF THE CABLES

\begin{tabular}{|l|l|}
\hline Core & $r=19.5 \mathrm{~mm}, \rho=3.365 E-8 \Omega . m$ \\
\hline Insulation & $t=18.25, \varepsilon_{r}=2.85$ \\
\hline Metallic sheath & $t=0.22 \mathrm{~mm}, \rho=1.718 E-8 \Omega . m$ \\
\hline Jacket & $t=4.53, \varepsilon_{r}=2.51$ \\
\hline
\end{tabular}

The time delays are calculated and presented in Table V. Three time delays are nearly equal. Then the six modes of propagation are lumped into four distinct modes, and then subjected to rational approximation. Table VI presents the fitting rms error of the modal propagation function using VFIT and RKFIT. The Table VI shows that a more accurate fitting is obtained with RKFIT using less number poles than VFIT.

TABLE V

CALCULATED TIME DELAYS (IN MILLISECOND)

\begin{tabular}{|l|l|l|l|l|l|}
\hline 0.803 & 1.945 & 6.414 & 1.684 & 1.684 & 1.684 \\
\hline
\end{tabular}

TABLE VI

MODAL PROPAGATION FUNCTION APPROXIMATION USING RKFIT AND VFIT

\begin{tabular}{ccccc}
\hline \multirow{2}{*}{ Mode } & \multicolumn{2}{c}{ RKFIT } & \multicolumn{2}{c}{ VFIT } \\
\cline { 2 - 5 } & RMS error & Model order & RMS error & Model order \\
\hline 1 & $9.05 \times 10^{-4}$ & 10 & $1.06 \times 10^{-3}$ & 16 \\
\hline 2 & $8.932 \times 10^{-4}$ & 17 & $1.16 \times 10^{-3}$ & 19 \\
\hline 3 & $2.52 \times 10^{-3}$ & 20 & $2.58 \times 10^{-3}$ & 20 \\
\hline 4 & $5.39 \times 10^{-3}$ & 6 & $4.73 \times 10^{-3}$ & 6 \\
\hline
\end{tabular}

The propagation function $H$ is fitted in the phase domain. Fig. 14 shows the deviation of the resulting fitting of the elements of $H$ for VFIT and RKFIT. Clearly, a better accuracy is obtained using RKFIT than VFIT. The maximum residue/pole ratio for RKFIT is 355.5 and for VFIT is 42.27 . The maximum residue/pole is lower than the critical threshold of 1000 [24].

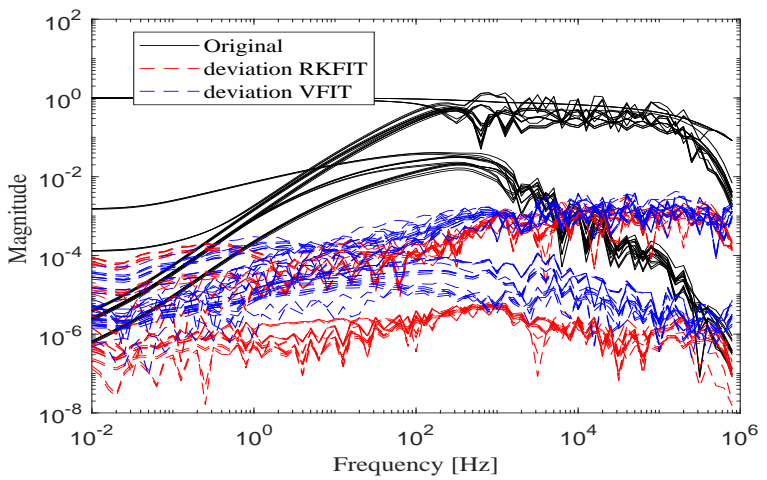

Fig. 14. Propagation function $\mathrm{H}$

The transient simulation is performed using the obtained model via RKFIT. The step voltage excitation is presented in Fig. 15, where the first core is energized with a unit step voltage at $t=0$. Fig. 16 shows the simulated receiving end voltage. The response is seen to be stable for the RKFIT model. The results are compared to accurate results obtained using the Numerical Inverse Laplace Transformation [25].

\section{DISCUSSION}

Till now, there are no comprehensive convergences analyses for VFIT and RKFIT. However, an example from [9], [26] was 


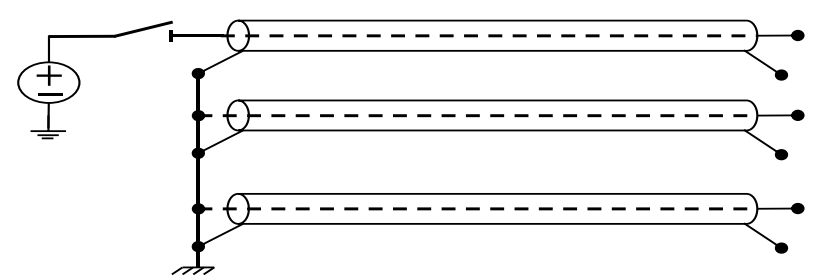

Fig. 15. Line energization by step voltage.

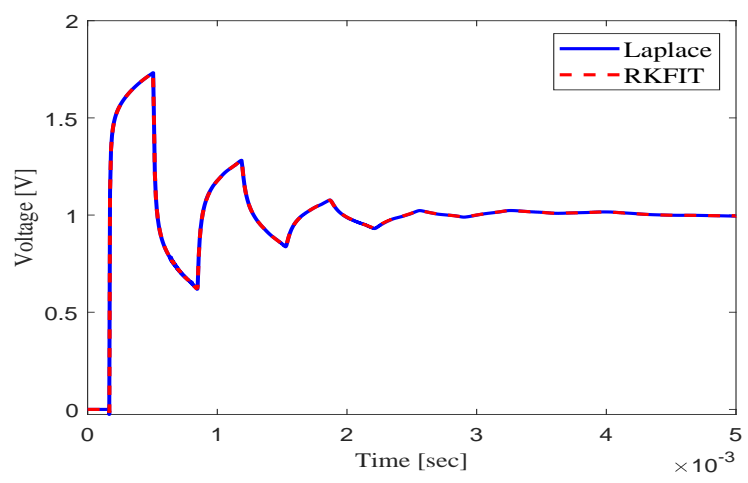

Fig. 16. Time domain simulation of unit step voltage response at the cable receiving end.

constructed where VFIT is repellent. Despite this example and Figure 6 in this work, VFIT is being successfully used for various problems. Nonetheless, RKFIT is based on a numerically well-defined computational scheme and have multiple advantages compared to VFIT. The proposed method determines the model order by itself and the reduction technique is implemented to reduce the model order if the fixed rms error allows it. The order reduction technique is not a post processing technique. It is one of the method proprieties and it is mathematically proven by theorem 6.1 in [9]. The final set of poles calculated by RKFIT is mathematically proven to be the optimal solution [9]. We observe this in the Haussdorff distance when it becomes equal to zero. However for VFIT, the Haussdorf distance decreases continuously and that means that the poles are slightly varying. The main drawback of RKFIT as VFIT is that they don't guarantee the passivity of the obtained model.

\section{CONCLUSION}

A new method for the rational approximation is studied and compared to well-known vector fitting. The proposed method is applied to transmission line modeling and specially in the universal line model. It was shown that this method delivers more accurate results than VFIT in the frequency domain and stable results in the time domain. The distinct advantage of the proposed method is the ease of the use by the engineers regarding the model order and the starting poles.

\section{REFERENCES}

[1] Adam Semlyen and A Dabuleanu. Fast and accurate switching transient calculations on transmission lines with ground return using recursive convolutions. IEEE Transactions on Power Apparatus and Systems, 94(2):561-571, 1975.

[2] Atef Morched, Bjorn Gustavsen, and Manoocher Tartibi. A universal model for accurate calculation of electromagnetic transients on overhead lines and underground cables. IEEE Transactions on Power Delivery, 14(3):1032-1038, 1999

[3] Bjem Gustavsen and Adam Semlyen. Simulation of transmission line transients using vector fitting and modal decomposition. IEEE Transactions on Power Delivery, 13(2):605-614, 1998.

[4] Bjorn Gustavsen and Adam Semlyen. Rational approximation of frequency domain responses by vector fitting. IEEE Transactions on power delivery, 14(3):1052-1061, 1999.

[5] Bjørn Gustavsen. Improving the pole relocating properties of vector fitting. IEEE Transactions on Power Delivery, 21(3):1587-1592, 2006.

[6] Abner Ramirez. Vector fitting-based calculation of frequency-dependent network equivalents by frequency partitioning and model-order reduction. IEEE Transactions on Power Delivery, 24(1):410-415, 2009.

[7] Miguel Cervantes Martinez, Ilhan Kocar, Jean Mahseredjian, and Abner Ramirez. Accurate and reduced order identification of propagation function for electromagnetic transient analysis of cables. IEEE Transactions on Power Delivery, 2019.

[8] Jesus Morales, Jean Mahseredjian, Abner Ramirez, Keyhan Sheshyekani, and Ilhan Kocar. A loewner/mpm-vf combined rational fitting approach. IEEE Transactions on Power Delivery, 2019.

[9] Mario Berljafa. Rational Krylov Decompositions: Theory and Applications. PhD thesis, The University of Manchester, 2017.

[10] Stefano Grivet-Talocia and Bjorn Gustavsen. Passive macromodeling: Theory and applications, volume 239. John Wiley \& Sons, 2015.

[11] Jörg Liesen and Zdenek Strakos. Krylov subspace methods: principles and analysis. Oxford University Press, 2013.

[12] Yousef Saad. Numerical methods for large eigenvalue problems: revised edition, volume 66. Siam, 2011.

[13] Mario Berljafa and Stefan Güttel. Generalized rational krylov decompositions with an application to rational approximation. SIAM Journal on Matrix Analysis and Applications, 36(2):894-916, 2015.

[14] Ion Victor Gosea and Stefan Güttel. Algorithms for the rational approximation of matrix-valued functions. arXiv preprint arXiv:2003.06410, 2020.

[15] B Gustavsen. Matrix fitting toolbox. Users guide and reference, SINTEF Energy Research, 2009.

[16] Younes Chahlaoui and Paul Van Dooren. A collection of benchmark examples for model reduction of linear time invariant dynamical systems. 2002.

[17] Nicholas J Higham, Gian Maria Negri Porzio, and Francoise Tisseur. An updated set of nonlinear eigenvalue problems. 2019.

[18] Ieee standard for binary floating-point arithmetic. ANSI/IEEE Std 754 1985, pages 1-20, 1985.

[19] Adam Semlyen and Bjørn Gustavsen. A half-size singularity test matrix for fast and reliable passivity assessment of rational models. IEEE Transactions on Power Delivery, 24(1):345-351, 2008.

[20] Hermann W Dommel. Electromagnetic Transients Program: Reference Manual:(EMTP theory book). Bonneville Power Administration, 1986.

[21] Bjørn Gustavsen. Optimal time delay extraction for transmission line modeling. IEEE Transactions on Power Delivery, 32(1):45-54, 2017.

[22] Ilhan Kocar and Jean Mahseredjian. New procedure for computation of time delays in propagation function fitting for transient modeling of cables. IEEE Transactions on Power Delivery, 31(2):613-621, 2016.

[23] Bjørn Gustavsen. Avoiding numerical instabilities in the universal line model by a two-segment interpolation scheme. IEEE Transactions on Power Delivery, 28(3):1643-1651, 2013.

[24] Ilhan Kocar, Jean Mahseredjian, and Guy Olivier. Improvement of numerical stability for the computation of transients in lines and cables. IEEE Transactions on Power Delivery, 25(2):1104-1111, 2010.

[25] Pablo Moreno and Abner Ramirez. Implementation of the numerical laplace transform: A review task force on frequency domain methods for emt studies, working group on modeling and analysis of system transients using digital simulation, general systems subcommittee, ieee power engineering society. IEEE Transactions on power delivery, 23(4):2599-2609, 2008

[26] Sanda Lefteriu and Athanasios C Antoulas. On the convergence of the vector-fitting algorithm. IEEE transactions on microwave theory and techniques, 61(4):1435-1443, 2013. 


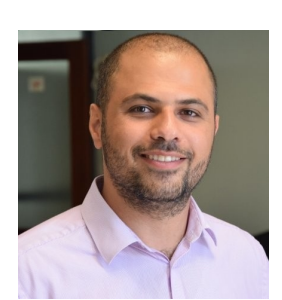

Amjad Mouhaidali has an electrical engineering diploma and the Ph.D. degree from Grenoble Alpes University (France) in 2015 and 2019, respectively. Currently, $\mathrm{He}$ is a research engineer based at SuperGrid institute, particularly in the HVDC cable systems and junctions research program. His research areas include the modeling of frequency-dependent and thermal effects in cable systems.

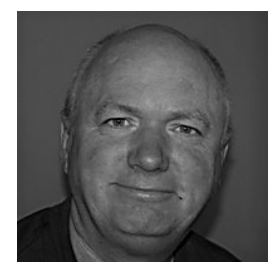

Damien Tromeur-Dervout received the Ph.D. degree in applied mathematics from University of Paris VI and ONERA, France, in 1993. Since 1994, He is with University Lyon 1, working on research and development activities related to numerical analysis of methods for high performances computing. In september 2015, he joined supergrid institute, program 1: Supergrid Architecture \& Systems, for developping numerical methods for the simulation of electrical networks .

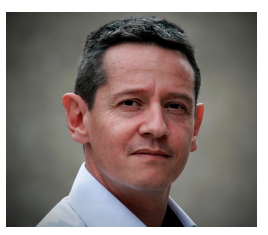

Olivier Chadebec was born in 1973, Sens, France. $\mathrm{He}$ received the Diploma in Electrical Engineering from the Grenoble Institute of Technology and the Ph.D. degrees from the Univ. Grenoble Alpes, France, in 1997 and 2001, respectively. He is currently CNRS senior researcher (directeur de recherche CNRS) and leads the "Models, Methods and Methodologies Applied to Electrical Engineering research group of G2ELab, Univ. Grenoble Alpes, France. He has been a visiting scientist at the Technology Centre of the Federal University of Santa Catarina, Brasil, in 2012-2013. His research activities focus on computational electromagnetics (finite element and integral methods), equivalent magnetic sources identification by solving inverse problems and low magnetic fields measurements. He has co-authored more than 250 papers published in international journals and conference proceedings.

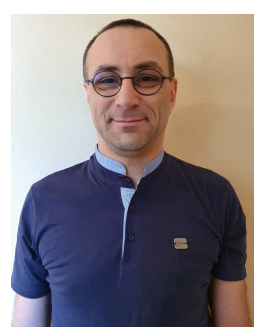

(without propagation).
Jean-Michel Guichon received the Diploma degree in electrical engineering and the Ph.D. degree from Grenoble Institute of Technology, Grenoble, France, in 1998 and 2001, respectively. $\mathrm{He}$ is an Associate Professor at the Universite Grenoble Alpes. He is has been with the Grenoble Electrical Engineering Laboratory (G2ELab), Grenoble, France, since 2003, working in the field of electromagnetic modeling for power electronics. He is focused in the development of numerical tools to compute the electromagnetic field and the electrical behavior in low frequency

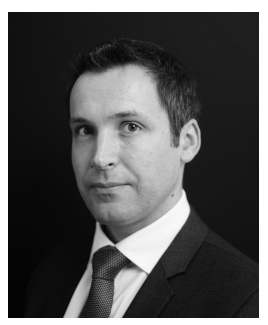

Sebastien Silvant graduated from the Ecole Polytechnique in Paris and from the EPFL in Lausanne in 2008. After leading innovation in high voltage circuit breakers, managing teams dedicated to numerical simulation software development, Sbastien has been responsible of a $R \& D$ group at Supergrid Institute, with the objective of developing models and applying real-time simulation to the control and protection of power systems and converters with a focus on HVDC. 